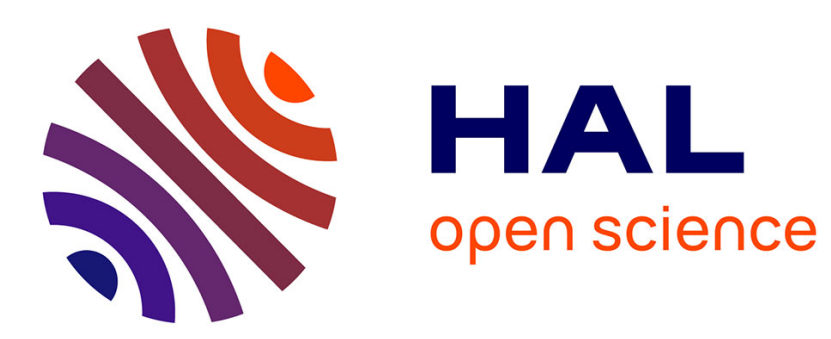

\title{
Nonlinear observer based on observable cascade form
} Mariem Sahnoun, Hassan Hammouri

\section{To cite this version:}

Mariem Sahnoun, Hassan Hammouri. Nonlinear observer based on observable cascade form. 2014. hal-00982014

\section{HAL Id: hal-00982014 https://hal.science/hal-00982014}

Preprint submitted on 23 Apr 2014

HAL is a multi-disciplinary open access archive for the deposit and dissemination of scientific research documents, whether they are published or not. The documents may come from teaching and research institutions in France or abroad, or from public or private research centers.
L'archive ouverte pluridisciplinaire HAL, est destinée au dépôt et à la diffusion de documents scientifiques de niveau recherche, publiés ou non, émanant des établissements d'enseignement et de recherche français ou étrangers, des laboratoires publics ou privés. 


\title{
Nonlinear observer based on observable cascade form
}

\author{
Mariem Sahnoun and Hassan Hammouri
}

\begin{abstract}
In this paper, the error observer linearization is extended to a class of observable cascade systems which contains state affine systems up to output injection. First, we give a theoretical result which states necessary and sufficient conditions. Next, we give an algorithm permitting to calculate a system of coordinates in which a nonlinear system takes the desired cascade observable form.
\end{abstract}

Index Terms-Nonlinear systems, output injection, nonlinear observer.

\section{INTRODUCTION}

The implementation of linear or nonlinear observers in control systems design, fault detection and other domains is well understood by now.

To design an observer for nonlinear systems, many approaches have been developed. Among them, the geometric approaches consist in characterizing nonlinear systems which can be transformed by a change of coordinates to a special class of systems for which a simple observer can be designed. The observer error linearization problem consists of transform a nonlinear system into a linear one plus a nonlinear term depending only on the known inputs and outputs. For such systems, a Luenberger observer can be designed. This problem has attracted a good deal of attention, since its formulation by [9] (see for instance [2], [3], [10]-[13]. Using immersion technics, an extension of this problem has been stated in [8] in the single output case. In the same spirit as for the error linearization problem, the authors in [4]-[7] characterized nonlinear systems which can be steered by a change of coordinates to state affine systems up to output injection. For these systems, a Kalman-like observer can be designed.

In this paper, we will characterize nonlinear systems which can be transformed by local coordinate systems into the following cascade form:

$$
\left\{\begin{array}{c}
\dot{z}=A(u) z+\psi(u, y) \\
\dot{\widetilde{z}}=\widetilde{A}(u) \widetilde{z}+\widetilde{\psi}(u, z, \widetilde{y}) \\
Y=\left(\begin{array}{c}
y \\
\widetilde{y}
\end{array}\right)=\left(\begin{array}{c}
C z \\
\widetilde{C} \widetilde{z}
\end{array}\right)
\end{array}\right.
$$

For these systems, an observer structure may take the

\footnotetext{
M. Sahnoun and $H$. Hammouri are with Université de Lyon, F-69622, Lyon, France; Université Lyon 1, Villeurbanne; CNRS, UMR 5007, LAGEP (Laboratoire d'Automatique et de Génie des Procédés). 43 bd du 11 novembre, 69100 Villeurbanne, France sahnoundlagep.univ-lyon1.fr, hammourielagep.univ-lyon 1 . fr
}

following form:

$$
\left\{\begin{array}{c}
\dot{\widehat{z}}=A(u) \widehat{z}+\psi(u, y)-S^{-1} C^{T} R(C \widehat{z}-y) \\
\dot{\widehat{\widetilde{z}}}=\widetilde{A}(u) \widetilde{\widetilde{z}}+\widetilde{\psi}(u, \widehat{z}, \widetilde{y})-\widetilde{S}^{-1} \widetilde{C}^{T} \widetilde{R}(\widetilde{C} \widetilde{z}-\widetilde{y}) \\
\dot{S}=-\theta S-A^{T}(u) S-S A(u)+C^{T} R C \\
\dot{\widetilde{S}}=-\widetilde{\theta} \widetilde{S}-\widetilde{A}^{T}(u) \widetilde{S}-\widetilde{S} \widetilde{A}(u)+\widetilde{C}^{T} \widetilde{R} \widetilde{C}
\end{array}\right.
$$

where $S(0), \widetilde{S}(0), R$ and $\widetilde{R}$ are symmetric positive definite matrices, $\theta>0, \widetilde{\theta}>0$ are parameters. The proof of the convergence of this observer has been stated in [1].

This paper is organized as follows:

In section II, the problem under consideration is formalized and an existence theorem is stated. In section III, an algorithm permitting to calculate a system of coordinates in which a nonlinear system takes the desired cascade form is proposed.

\section{PRELIminary RESUltS AND EXISTENCE THEOREM}

\section{A. Preliminary results}

For the sake of simplicity, we only consider the case where the outputs $y$ and $\tilde{y}$ are scalars. The following classes of nonlinear systems will be considered:

$$
\left\{\begin{array}{l}
\dot{x}=f(u, x) \\
y=h(x) \\
\widetilde{y}=\widetilde{h}(x)
\end{array}\right.
$$

where $x \in \mathbb{R}^{n}$, the input $u(t) \in \mathbb{R}^{m}$ and the outputs $y(t)$ and $\widetilde{y}(t)$ are belong to $\mathbb{R}$. $f, h$ and $\widetilde{h}$ are assumed to be of class $\mathscr{C}^{\infty}$.

We adopt the following definition.

Definition 1: System (1) is said to be cascadeobservable, if system (1) together with its associated reduced system in $z$ are observable.

The following geometric notions will be used in the sequel. In the system of coordinates $\left(x_{1}, \ldots, x_{n}\right)$, let $X=\sum_{i=1}^{n} \alpha_{i} \frac{\partial}{\partial x_{i}}$ be a vector field and let $\omega=\sum_{i=1}^{n} a_{i} d x_{i}$ a one-differential form, then the following operations will be considered:

- Lie derivative action: $L_{X}(\omega)=\sum_{i=1}^{n} \alpha_{i} L_{X}\left(a_{i}\right) d x_{i}+$ $\sum_{i=1}^{n} a_{i} d \alpha_{i}$

- The duality product: $\omega(X)=\sum_{i=1}^{n} \alpha_{i} a_{i}$

The above duality product can be extended to $k$ differential forms as follow:

If $\omega=\sum_{1 \leq i_{1}<\ldots<i_{k} \leq n} a_{\left(i_{1}, \ldots, i_{k}\right)} d x_{i_{1}} \wedge \ldots \wedge d x_{i_{k}}$ is a $k$-differential form and $X=\left(X_{1}, \ldots, X_{k}\right)$ is a $k$ tuple of vector fields, with $X_{i}=\sum_{l=1}^{n} \alpha_{i l} \frac{\partial}{\partial x_{l}}$, then 


$$
\omega(X)=\sum_{1 \leq i_{1}<\ldots<i_{k} \leq n} a_{\left(i_{1}, \ldots, i_{k}\right)}\left|\begin{array}{ccc}
\alpha_{1 i_{1}} & \ldots & \alpha_{k i_{1}} \\
\ldots & \ldots & \ldots \\
\alpha_{1 i_{k}} & \ldots & \alpha_{k i_{k}}
\end{array}\right| .
$$

- Inner product: Let $X=\left(X_{1}, \ldots, X_{l}\right)$ be a $l$-tuple of vector fields, with $l \leq k$. Then $i_{X}(\omega)$ is the $(k-l)$ differential form defined by:

$$
i_{X}(\omega)\left(Y_{1}, \ldots, Y_{k-l}\right)=\omega\left(X_{1}, \ldots, X_{l}, Y_{1}, \ldots, Y_{k-l}\right) .
$$

In particular, if $k=l$, then $i_{X}(\omega)$ is a function (a 0 -differential form).

Let $f_{u}$ be the vector field defined by $f_{u}(x)=f(u, x)$ and let $X$ be a vector field on $\mathbb{R}^{n}$. We define the family of real vector spaces $\Omega_{k}^{X}$ of 2-differential forms as follows:

- $\Omega_{0}^{X}=0$ and $\Omega_{1}^{X}=\operatorname{Span}\left\{d L_{f_{u}}(h) \wedge d h ; u \in \mathbb{R}^{m}\right\}$. Noticing that these two spaces do not depend on $X$,

- for $k \geq 1$, we set $\Omega_{k+1}^{X}=\operatorname{Span}\left\{L_{f_{u}}\left(i_{X}(\omega)\right) \wedge d h ; u \in\right.$ $\left.\mathbb{R}^{m} ; \omega \in \Omega_{k}^{X}\right\}+\Omega_{k}^{X}$.

Now setting $\pi=d \varphi_{1} \wedge \ldots \wedge d \varphi_{q}$, where $\varphi_{k}$ are $\mathscr{C}^{\infty}$ functions, and let $\widetilde{X}=\left(\widetilde{X}_{1}, \ldots, \widetilde{X}_{q+1}\right)$ be a $(q+1)$-tuple of vector fields. As above, we define the vector spaces $\Omega_{k, \pi}^{\widetilde{X}}$ of $(q+2)$ differential forms as follows:

- $\Omega_{0, \pi}^{\widetilde{X}}=0$ and $\Omega_{1, \pi}^{\widetilde{X}}=\operatorname{Span}\left\{d L_{f_{u}}(\widetilde{h}) \wedge d \widetilde{h} \wedge \pi ; u \in \mathbb{R}^{m}\right\}$,

- for $k \geq 1, \Omega_{k+1, \pi}^{\widetilde{X}}=\operatorname{Span}\left\{L_{f_{u}}\left(i_{\widetilde{X}}(\widetilde{\omega})\right) \wedge d \widetilde{h} \wedge \pi ; u \in\right.$ $\left.\mathbb{R}^{m} ; \widetilde{\omega} \in \Omega_{k, \pi}^{\widetilde{X}}\right\}+\Omega_{k, \pi}^{\widetilde{X}}$.

\section{B. Existence theorem}

In the single output case (see [4], [6]), ( [5] for the the multi-output case) the authors gave necessary and sufficient conditions under which nonlinear systems can be transformed in a state affine system up to output injection.

The following theorem states an existence theorem which extends those stated in [4], [5]:

\section{Theorem 1:}

Observable system (3) can be transformed by a local change of coordinates around some $x^{0} \in \mathbb{R}^{n}$ to a cascadeobservable system (1) in which $C$ and $\widetilde{C}$ are of rank 1, if and only if, the following conditions hold on some neighborhood of $x^{0}$ :

1) It exists a vector field $X$ satisfying the following conditions:

1-i) $L_{X}(h)=1$.

1-ii) The algebraic sum $\Omega^{X}=\sum_{k \geq 1} \Omega_{k}^{X}$ is a real vector space of dimension $q-1$.

1-iii) For every $\omega \in \Omega^{X}, d\left(i_{X}(\omega)\right)=0$.

1-iv) The dimension of $\left.\left[\wedge^{q-1}\left(i_{X}\left(\Omega^{X}\right)\right) \wedge d h\right]\right|_{x^{0}}$ is equal to 1 , where $\left.\left[\wedge^{q-1}\left(i_{X}\left(\Omega^{X}\right)\right) \wedge d h\right]\right|_{x^{0}}=\left\{i_{X}\left(\omega_{1}\right) \wedge \ldots \wedge\right.$ $\left.i_{X}\left(\omega_{q-1}\right) \wedge d h\left(x^{0}\right) ; \quad \omega_{i} \in \Omega^{X}, 1 \leq i \leq q-1\right\}$.

2) Consider the following functions $\varphi_{1}, \ldots, \varphi_{q+1}$ defined by:

$$
\begin{aligned}
& \varphi_{1}=h \\
& \varphi_{q+1}=\widetilde{h} \\
& \left(d \varphi_{1}, \ldots, d \varphi_{q}\right) \text { forms a basis of } i_{X}\left(\Omega^{X}\right)+\mathbb{R} d h
\end{aligned}
$$

Setting $\pi=d \varphi_{1} \wedge \ldots \wedge d \varphi_{q}$, then there exists a $(q+1)$ tuple of vector fields $\widetilde{X}=\left(\widetilde{X}_{1}, \ldots, \widetilde{X}_{q+1}\right)$ satisfying the following conditions on some neighborhood of $x^{0}$ :

2-i) $L_{\widetilde{X}_{i}}\left(\varphi_{j}\right)=\delta_{i j}$, where $\delta_{i j}=1$ if $i=j$ and 0 otherwise.

2-ii) The algebraic sum $\Omega_{\pi}^{\widetilde{X}}=\sum_{k \geq 1} \Omega_{k, \pi}^{\widetilde{X}}$ is a real vector space of dimension $n-q-1$.

2-iii) For every $\widetilde{\omega} \in \Omega_{\pi}^{\widetilde{X}}, d\left(i_{\widetilde{X}}(\widetilde{\omega})\right)=0$.

2-iv) The dimension of $\left[\wedge^{n-q-1}\left(i_{\widetilde{X}}\left(\Omega_{\pi}^{\widetilde{X}}\right)\right) \wedge d \varphi_{1} \wedge \ldots \wedge\right.$ $\left.d \varphi_{q+1}\right]\left.\right|_{x^{0}}$ is equal to 1 .

The proof of theorem 1 can be obtained by following the same approach as the one proposed in the works [4], [5].

The outline of the proof is summarized as follows:

1) Sufficient condition: $i_{X}\left(\Omega^{X}\right)$ and $i_{\widetilde{X}}\left(\Omega_{\pi}^{\widetilde{X}}\right)$ are vector spaces of dimension $q-1$ and $n-q-1$ respectively, and $\left(i_{X}\left(\omega_{1}\right), \ldots, i_{X}\left(\omega_{q-1}\right)\right), \quad\left(i_{\widetilde{X}}\left(\widetilde{\omega}_{1}\right), \ldots, i_{\widetilde{X}}\left(\widetilde{\omega}_{n-q-1}\right)\right)$ are their respective bases. Setting $d z_{1}=d h, d z_{i}=$ $i_{X}\left(\omega_{i+1}\right), d \widetilde{z}_{1}=d \widetilde{h}$ and $d \widetilde{z}_{i}=i_{\widetilde{X}}\left(\widetilde{\omega}_{i+1}\right)$. It can be shown that $L_{f_{u}}\left(z_{i}\right)=\sum_{j=2}^{q} a_{i j}(u) z_{j}+\psi_{i}\left(u, z_{1}\right)$ and $L_{f_{u}}\left(\widetilde{z}_{i}\right)=$ $\sum_{j=2}^{n-q} \widetilde{a}_{i j}(u) \widetilde{z}_{j}+\psi_{i}\left(u, z, \widetilde{z}_{1}\right)$. Consequently, in the $(z, \widetilde{z})$ system of coordinates system (3) takes the cascade form (1).

2) Necessary condition: Since conditions 1), 2) of theorem 1 are intrinsic (they do not depend on the system of coordinates), it suffices to show them for the cascade observable system (1). After a simple linear change of coordinates, we can assume that $y=C z=z_{1}$ and $\widetilde{y}=$ $\widetilde{C} \widetilde{z}=\widetilde{z}_{1}$, and it can be shown that $X=\frac{\partial}{\partial z_{1}}$ and $\widetilde{X}=$ $\left(\widetilde{X}_{1}, \ldots, \widetilde{X}_{q+1}\right)=\left(\frac{\partial}{\partial z_{1}}, \ldots, \frac{\partial}{\partial z_{q}}, \frac{\partial}{\partial \widetilde{z}_{1}}\right)$ satisfy conditions 1) and 2) of theorem 1.

In the following, we focus on the development of an algorithm permitting to calculate vector fields $X, \widetilde{X}_{1}, \ldots, \widetilde{X}_{q+1}$ which meet conditions 1) and 2) of theorem 1.

\section{Procedure of CAlculation of Vector Fields $X, \widetilde{X}_{1}, \ldots, \widetilde{X}_{q+1}$}

\section{A. Preliminary results}

The following notations will be used in the sequel:

- Let $V$ be a vector space, and $W$ a subspace of $V$, then for $\xi, \xi^{\prime} \in V$, the notation $\xi=\xi^{\prime}$ modulo $(W)$ means that $\xi=\xi^{\prime}+w$, for some $w \in W$.

- Setting $\mathscr{F}$ (resp. $\mathscr{V}$ ) to be a set of one-differential form (resp. of vector fields). $D=\operatorname{Span}(\mathscr{F})$ (resp. $\Delta=$ $\operatorname{Span}(\mathscr{V})$ ) will denote the co-distribution (resp. the distribution) spanned by $\mathscr{F}$ (resp. by $\mathscr{V}$ ).

- The orthogonal of a co-distribution $D$ is the distribution $\Delta=\operatorname{Ker}(D)=\operatorname{Span}(\{X ; \omega(X)=0, \forall \omega \in \mathscr{F}\})$, where $\omega(X)$ is the duality product between one-form and vector fields. In particular, if $\mathscr{F}$ is spanned by a family of one-exact form $\{d \varphi ; \varphi \in \widetilde{\mathscr{F}}\}$, then $\Delta=\operatorname{Ker}(D)$ 
is the distribution spanned by the set of vector fields $\left\{X ; L_{X}(\varphi)=0, \forall \varphi \in \widetilde{\mathscr{F}}\right\}$.

- Let $D, D^{\prime}$ be two co-distributions, with $D^{\prime} \subset D$, then the quotient $D / D^{\prime}$ will denote the set of equivalent class of differential forms $[\omega]=\omega+D^{\prime}=\left\{\omega+\omega^{\prime} ; \omega^{\prime} \in D^{\prime}\right\}$, where $\omega \in D$. Similarly, if $\Delta \subset \Delta^{\prime}$ are two distributions, elements of the quotient $\Delta^{\prime} / \Delta$ will be denoted by $[X]=$ $X+\Delta$ where $X \in \Delta^{\prime}$.

If $[\omega] \in D / D^{\prime}$ and $\chi \in D$ such that $[\omega]=[\chi]$, then we set $\omega=\chi$ modulo $\left(D^{\prime}\right)$.

Finally, if $X, Z$ are two vector fields, $[X, Z]$ will denote the Lie bracket of these vector fields.

The following flag of co-distributions and distributions will be considered:

$$
\begin{aligned}
& D_{0} \subset \ldots \subset D_{k} \subset \ldots \\
& \Delta_{0} \supset \ldots \supset \Delta_{k} \supset \ldots \\
& \widetilde{D}_{0} \subset \ldots \subset \widetilde{D}_{k} \subset \ldots \\
& \widetilde{\Delta}_{0} \supset \ldots \supset \widetilde{\Delta}_{k} \supset \ldots
\end{aligned}
$$

Where,

- $D_{0}=0$ the null co-distribution, $D_{1}=\operatorname{Span}(\{d h\})$, by induction $D_{k+1}=D_{k}+\operatorname{Span}\left(\left\{d L_{f_{u_{k}}} \ldots L_{f_{u_{1}}}(h) ; u_{1}, \ldots\right.\right.$, $\left.\left.u_{k} \in \mathbb{R}^{m}\right\}\right)$, and $D_{\sharp}=\sum_{k \geq 1} D_{k}$.

- $\widetilde{D}_{0}=D_{\sharp}, \widetilde{D}_{1}=\widetilde{D}_{0}+\operatorname{Span}(\{d \widetilde{h}\})$, for $k \geq 1, \widetilde{D}_{k+1}=$ $\widetilde{D}_{k}+\operatorname{Span}\left(\left\{d L_{f_{u_{k}}} \ldots L_{f_{u_{1}}}(\widetilde{h}) ; u_{1}, \ldots, u_{k} \in \mathbb{R}^{m}\right\}\right)$, and $\widetilde{D}_{\sharp}=\sum_{k \geq 1} \widetilde{D}_{k}$.

- $\Delta_{k}=\operatorname{Ker}\left(D_{k}\right)$, and $\Delta_{\sharp}=\operatorname{Ker}\left(D_{\sharp}\right)$.

- $\widetilde{\Delta}_{k}=\operatorname{Ker}\left(\widetilde{D}_{k}\right)$, and $\widetilde{\Delta}_{\sharp}=\operatorname{Ker}\left(\widetilde{D}_{\sharp}\right)$.

- The quotient co-distribution $D_{k} / D_{k-1}\left(\operatorname{resp} . \widetilde{D}_{k} / \widetilde{D}_{k-1}\right)$ is the dual of the quotient distribution $\Delta_{k-1} / \Delta_{k}$ (resp. $\left.\widetilde{\Delta}_{k-1} / \widetilde{\Delta}_{k}\right)$. The duality product $[\omega]([X])=\omega(X)$ is well defined.

In the two following claims, $f_{u}=\sum_{i=1}^{q}\left(A_{i}(u) z+\psi_{i}(u, y)\right) \frac{\partial}{\partial z_{i}}+$ $\sum_{i=1}^{n-q}\left(\widetilde{A}_{i}(u) \widetilde{z}+\widetilde{\psi}_{i}(u, z, \widetilde{y})\right) \frac{\partial}{\partial \widetilde{z}_{i}}$, and the outputs $h, \widetilde{h}$ are respectively $y=C z=z_{1}, \widetilde{y}=\widetilde{C} \widetilde{z}=\widetilde{z}_{1}$.

Considering the rings $\mathscr{H}_{k}, \widetilde{\mathscr{H}}_{k}$ such that:

- $\mathscr{H}_{0}=\mathscr{C}^{\infty}\left\{z_{1}\right\}$ (resp. $\widetilde{\mathscr{H}_{0}}=\mathscr{C}^{\infty}\left\{z_{1}, \ldots, z_{q}, \widetilde{z}_{1}\right\}$ ) is the ring of $\mathscr{C}^{\infty}$-functions $\varphi\left(z_{1}\right)$ (resp. $\varphi\left(z_{1}, \ldots, z_{q}, \widetilde{z}_{1}\right)$ ).

- $\mathscr{C}^{\infty}\{z\}$ (resp. $\mathscr{C}^{\infty}\{z, \widetilde{z}\}$ ) denotes the ring of $\mathscr{C}^{\infty}$ functions $\varphi\left(z_{1}, \ldots, z_{q}\right)\left(\operatorname{resp} . \varphi\left(z_{1}, \ldots, z_{q}, \widetilde{z}_{1}, \ldots, \widetilde{z}_{n-q}\right)\right)$. Then for $k \geq 1, \mathscr{H}_{k}$ (resp. $\widetilde{\mathscr{H}}_{k}$ ) is the smallest subring of $\mathscr{C}^{\infty}\{z\}$ (resp. of $\mathscr{C}^{\infty}\{z, \widetilde{z}\}$ ) containing $\mathscr{H}_{k-1} \cup$ $\left\{\underset{\widetilde{C}}{C A}\left(u_{1}\right) \ldots \underset{\sim}{A}\left(u_{k}\right) z ; \quad u_{1}, \ldots, u_{k} \in \mathbb{R}^{m}\right\} \quad\left(\right.$ resp. $\widetilde{\mathscr{H}}_{k-1} \cup$ $\left.\left\{\widetilde{C} \widetilde{A}\left(u_{1}\right) \ldots \widetilde{A}\left(u_{k}\right) \widetilde{z} ; u_{1}, \ldots, u_{k} \in \mathbb{R}^{m}\right\}\right)$.

Then we have:

Claim 1:

i) $L_{f_{u_{k}}} \ldots L_{f_{u_{1}}}(C z)=C A\left(u_{1}\right) \ldots A\left(u_{k}\right) z$ modulo $\left(\mathscr{H}_{k-1}\right)$.

ii) $\left.L_{f_{u_{k}}} \ldots L_{f_{u_{1}}}(\widetilde{C Z})\right)=\widetilde{C} \widetilde{A}\left(u_{1}\right) \ldots \widetilde{A}\left(u_{k}\right) \widetilde{z}$ modulo $\left(\widetilde{\mathscr{H}_{k-1}}\right)$.

The following claim can be deduced from the above one.

\section{Claim 2:}

- The flags of co-distributions $D_{0} \subset \ldots \subset D_{k} \subset \ldots$; $\widetilde{D}_{0} / D_{\sharp} \subset \ldots \subset \widetilde{D}_{k} / D_{\sharp} \subset \ldots$ are of constant dimensions and defined as follows:

a) $D_{1}=\operatorname{Span}(d C z)$, and for $k \geq 2, D_{k}$ is spanned by the set of one-forms $\{d C z\} \cup\left\{d C A\left(u_{1}\right) \ldots A\left(u_{l}\right) z ; 1 \leq l \leq\right.$ $\left.k-1, u_{j} \in \mathbb{R}^{m}\right\}$.

b) Similarly, $\widetilde{D}_{1} / D_{\sharp}$ can be identified with the codistribution $\operatorname{Span}(d \widetilde{C} \widetilde{Z})$, and for $k \geq 2, \widetilde{D}_{k} / D_{\sharp}$ is isomorphic to the co-distribution spanned by the set of oneforms $\{d \widetilde{C} \widetilde{z}\} \cup\left\{d \widetilde{C} \widetilde{A}\left(u_{1}\right) \ldots \widetilde{A}\left(u_{l}\right) \widetilde{z} ; 1 \leq l \leq k-1, u_{j} \in\right.$ $\left.\mathbb{R}^{m}\right\}$.

- System (1) is cascade observable iff: $\operatorname{dim} D_{\sharp}=q$ ( $q$ is the dimension of the $z$-space), and $\operatorname{dim} \widetilde{D}_{\sharp} / D_{\sharp}=n-q(n-q$ is the dimension of $\widetilde{z}$-space).

In the sequel, we set $v(\operatorname{resp} \widetilde{v})$ to be the smallest integer such that $D_{v}=D_{\sharp}\left(\operatorname{resp} . \widetilde{D}_{\widetilde{v}} / D_{\sharp}=\widetilde{D}_{\sharp} / D_{\sharp}\right)$ :

$$
\begin{aligned}
& D_{0} \subset \ldots \subset D_{v}=D_{v+1} \\
& \widetilde{D}_{0} / D_{\sharp} \subset \ldots \subset \widetilde{D}_{\widetilde{v}} / D_{\sharp}=\widetilde{D}_{\widetilde{v}+1} / D_{\sharp}
\end{aligned}
$$

This subsection will be ended by the two following technical results:

\section{Lemma 1:}

If $d \varphi \in D_{k-1}$ (resp. $\left.d \widetilde{\varphi} \in \widetilde{D}_{k-1}\right)$ and $X \in \Delta_{k-1}$ (resp. $\widetilde{X} \in$ $\left.\widetilde{\Delta}_{k-1}\right)$, then $d \varphi\left(\left[f_{u}, X\right]\right)=-d\left(L_{f_{u}}(\varphi)\right)(X)=-L_{X}\left(L_{f_{u}}(\varphi)\right)$ (resp. $\left.d \varphi\left(\left[f_{u}, \widetilde{X}\right]\right)=-d\left(L_{f_{u}}(\widetilde{\varphi})\right)(\widetilde{X})=-L_{\widetilde{X}}\left(L_{f_{u}}(\widetilde{\varphi})\right)\right)$.

\section{Proof of lemma 1.}

Let $d \varphi \in D_{k-1}$ and $X \in \Delta_{k-1}$, the equality $d \varphi\left(\left[f_{u}, X\right]\right)=$ $-d\left(L_{f_{u}}(\varphi)\right)(X)$ follows from the following facts:

$$
\begin{aligned}
\text { - } & d \varphi\left(\left[f_{u}, X\right]\right)=L_{f_{u}}\left(L_{X}(\varphi)\right)-L_{X}\left(L_{f_{u}}(\varphi)\right) \\
& =d\left(L_{X}(\varphi)\right)\left(f_{u}\right)-d\left(L_{f_{u}}(\varphi)\right)(X), \\
\text { - } & X \in \Delta_{k} \subset \Delta_{k-1}=\operatorname{Ker}\left(D_{k-1}\right), \\
\text { - } & L_{X}(\varphi)=d \varphi(X)=0
\end{aligned}
$$

Similar argument can be used to prove $d \varphi\left(\left[f_{u}, \widetilde{X}\right]\right)=$ $-d\left(L_{f_{u}}(\widetilde{\varphi})\right)(\widetilde{X})$.

\section{Claim 3:}

Let $Z=\left(Z_{1}, \ldots, Z_{k}\right)$ be a $k$-tuple of vector fields, let $g, \varphi_{1}, \ldots, \varphi_{k}$ be $\mathscr{C}^{\infty}$-functions such that $d \varphi_{1} \wedge \ldots \wedge d \varphi_{k}$ is nowhere vanish and that $L_{Z_{j}}\left(\varphi_{i}\right)=\delta_{i j}$, then:

$i_{Z}\left(d g \wedge d \varphi_{1} \wedge \ldots \wedge d \varphi_{k}\right)=d g-\sum_{j=1}^{k} L_{Z_{j}}(g) d \varphi_{j}$.

More precisely, we have:

$i_{Z}\left(d g \wedge d \varphi_{1} \wedge \ldots \wedge d \varphi_{k}\right)=(-1)^{q}\left(d g-\sum_{j=1}^{k} L_{Z_{j}}(g) d \varphi_{j}\right)$.

\section{B. Algorithm}

In this subsection, we will give an algorithm permitting to calculate the vector fields $X, \widetilde{X}_{1}, \ldots, \widetilde{X}_{q+1}$, which meet conditions of theorem 1 . This algorithm will be obtained in three steps:

1) The first step consists to calculate $X$ using only $f(u, x)$ and $h(x)$. 
2) The knowledge of $f(u, x), h, \widetilde{h}(x)$ and $X$ allows to calculate $\widetilde{X}_{q+1}$.

3) Finally, $\widetilde{X}_{1}, \ldots, \widetilde{X}_{q}$ can be computed based on the knowledge of $f(u, x), h, \widetilde{h}(x), X$ and $\widetilde{X}_{q+1}$.

Assuming that the flags of co-distributions:

$$
\begin{aligned}
& 0=D_{0} \subset \ldots \subset D_{v}=D_{v+1} \\
& 0=\widetilde{D}_{0} / D_{v} \subset \ldots \subset \widetilde{D}_{\widetilde{v}} / D_{v}=\widetilde{D}_{\widetilde{v}+1} / D_{v}
\end{aligned}
$$

are of constant dimensions and that $\operatorname{dim}\left(D_{v}\right)=q$, $\operatorname{dim}\left(\widetilde{D}_{\widetilde{v}} / D_{v}\right)=n-q$.

For $k \geq 1$, we define the bases $B_{k}$ and $\widetilde{B}_{k}$ of $D_{k} / D_{k-1}$ and $\widetilde{D}_{k} / \widetilde{D}_{k-1}$ as follows:

$$
\begin{aligned}
& B_{1}=\{[d h]\}, \widetilde{B}_{1}=\{[d \tilde{h}]\} \\
& \text { for } k \geq 2: \\
& B_{k}=\left\{\left[d\left(L_{f_{u_{k-1}}} \ldots L_{f_{u_{1}}}(h)\right)\right] ; \quad\left(u_{1}, \ldots, u_{k-1}\right) \in \mathscr{U}_{k-1}\right\} \\
& \widetilde{B}_{k}=\left\{\left[d\left(L_{\widetilde{u}_{\widetilde{u}-1}} \ldots L_{\widetilde{u}_{\widetilde{u}_{1}}}(\tilde{h})\right)\right] ;\left(\widetilde{u}_{1}, \ldots, \widetilde{u}_{k-1}\right) \in \widetilde{\mathscr{U}}_{k-1}\right\}
\end{aligned}
$$

for some subsets $\mathscr{U}_{k-1}$ and $\widetilde{\mathscr{U}_{k-1}}$ of $\left(\mathbb{R}^{m}\right)^{k-1}$.

The symbol $[()$.$] stands for the equivalent class of (.).$

Now, let $B_{v}^{*}, \widetilde{B}_{\widetilde{v}}^{*}$ be the respective dual bases of $B_{v}$ and $\widetilde{B}_{\widetilde{v}}$ $\left(B_{v}^{*}, \widetilde{B}_{\widetilde{v}}^{*}\right.$ are bases of $\Delta_{v-1} / \Delta_{v}$ and $\left.\widetilde{\Delta}_{\widetilde{v}-1} / \widetilde{\Delta}_{\widetilde{v}}\right)$, the following vector fields will be required in theorem 2 below :

- The vector fields $\left[Z_{u_{1} \ldots u_{v-1}}\right],\left[\widetilde{Z}_{\widetilde{u}_{1} \ldots \widetilde{u}_{\widetilde{v}-1}}\right]$ :

Let $\left(u_{1}, \ldots, u_{v-1}\right)$, (resp. $\left.\left(\widetilde{u}_{1}, \ldots, \widetilde{u}_{\widetilde{v}-1}\right)\right)$ be fixed elements of $\mathscr{U}_{v-1}$ (resp. of $\widetilde{\mathscr{U}}_{\tilde{v}-1}$ ), then $[Y]=\left[Z_{u_{1} \ldots u_{v-1}}\right]$ (resp. $[\tilde{Y}]=$ $\left[\widetilde{Z}_{\widetilde{u}_{1} \ldots \widetilde{u}_{\widetilde{v}-1}}\right]$ ) is the element of $B_{v}^{*}\left(\right.$ resp. of $\left.\widetilde{B}_{\widetilde{v}}^{*}\right)$ defined by:

for $\left(v_{1}, \ldots, v_{v-1}\right) \in \mathscr{U}_{v-1}, \quad d\left(L_{f_{v_{v-1}}} \ldots L_{f_{v_{1}}}(h)\right)(Y)=1$, if $\left(u_{1}, \ldots, u_{v-1}\right)=\left(v_{1}, \ldots, v_{v-1}\right)$, and 0 otherwise for $\left(\widetilde{v}_{1}, \ldots, \widetilde{v}_{\widetilde{v}-1}\right) \in \widetilde{\mathscr{U}}_{\widetilde{v}-1}, \quad d\left(L_{\tilde{v}_{\widetilde{v}-1}} \ldots L_{\widetilde{v}_{\widetilde{v}}}(\widetilde{h})\right)(\widetilde{Y})=1$, if $\left(\widetilde{u}_{1}, \ldots, \widetilde{u}_{\widetilde{v}-1}\right)=\left(\widetilde{v}_{1}, \ldots, \widetilde{v}_{\widetilde{v}-1}\right)$, and 0 otherwise

- The vector fields $\left[Y^{u_{1} \ldots u_{v-1}}\right],\left[\widetilde{Y}^{\widetilde{u}_{1} \ldots \widetilde{u}_{\widetilde{v}-1}}\right]$ : Setting $[Y]=\left[Z_{u_{1} \ldots u_{v-1}}\right]$ and $[\widetilde{Y}]=\left[\widetilde{Z}_{\widetilde{u}_{1} \ldots \widetilde{u}_{\widetilde{v}-1}}\right]$, then:

$$
\begin{aligned}
Y^{u_{1} \ldots u_{v-1}} & \left.=\left[f_{u_{v-1}},\left[\ldots,\left[f_{u_{1}}, Y\right] \ldots\right]\right]\right] \\
\widetilde{Y}^{\widetilde{u}_{1} \ldots \widetilde{u}_{\widetilde{v}-1}} & \left.=\left[f_{\widetilde{u}_{\widetilde{v}-1}},\left[\ldots,\left[f_{\widetilde{u}_{1}}, Y\right] \ldots\right]\right]\right]
\end{aligned}
$$

In order to state lemma 2 below, the following notations will be required:

- Let $\left(d \varphi_{1}, \ldots, d \varphi_{q}\right)$ be a basis of $D_{v}$ and $d \varphi_{q+1}=d \widetilde{h}$.

- Setting $\tilde{\pi}=d \varphi_{1} \wedge \ldots \wedge d \varphi_{q+1}$.

- Let $\widetilde{X}=\left(\widetilde{X}_{1}, \ldots, \widetilde{X}_{q+1}\right)$ be a $(q+1)$-tuple of vector fields satisfying $L_{\widetilde{X}_{i}}\left(\varphi_{j}\right)=\delta_{i j}$.

- For $\widetilde{u}_{1} \in{\widetilde{\mathscr{U}_{1}}}_{1}$, we set $\widetilde{\omega}_{\widetilde{u}_{1}}=d L_{\widetilde{u}_{1}}(\widetilde{h}) \wedge \tilde{\pi}$.

- For $k \geq 2$ and $\left(\widetilde{u}_{1}, \ldots, \widetilde{u}_{k}\right) \in \widetilde{\mathscr{U}}_{k}$, we set $\widetilde{\omega}_{\widetilde{u}_{1} \ldots \widetilde{u}_{k}}=$ $L_{\widetilde{u}_{k}}\left(i_{\widetilde{X}}\left(\widetilde{\omega}_{\widetilde{u}_{1} \ldots \widetilde{u}_{k-1}}\right)\right) \wedge \tilde{\pi}$.

Thus we have:

Lemma 2:

For $1 \leq k \leq \widetilde{v}-1$; for every $\left(\widetilde{u}_{1}, \ldots, \widetilde{u}_{k}\right) \in \widetilde{\mathscr{U}_{k}}$ the following properties hold:

$$
\begin{aligned}
& \widetilde{\omega}_{\widetilde{u}_{1} \ldots \widetilde{u}_{k}}=d L_{\widetilde{u}_{\widetilde{u}_{k}}} \ldots L_{{\widetilde{u}}_{1}}(\widetilde{h}) \wedge \tilde{\pi} \\
& +\sum_{l=1}^{k-1} \sum_{\left(\widetilde{u}_{1}, \ldots, \widetilde{u}_{l}\right) \in \widetilde{\mathscr{U}}_{l}} g_{\widetilde{u}_{1} \ldots \widetilde{u}_{l}}(x) d L_{{\widetilde{u_{l}}}_{l}} \ldots L_{{\widetilde{u}}_{1}}(\widetilde{h}) \wedge \tilde{\pi}
\end{aligned}
$$

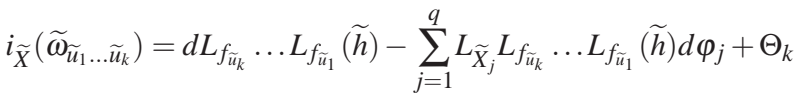

$$
\begin{aligned}
& \Theta_{k}=\widetilde{\Theta}_{k}-\sum_{j=1}^{q} \sum_{l=1}^{k-1} \sum_{\left(\widetilde{u}_{1}, \ldots, \widetilde{u}_{l}\right) \in \widetilde{\mathscr{U}}_{l}} g_{\widetilde{u}_{1} \ldots \widetilde{u}_{l}}(x) L_{\widetilde{X}_{j}} L_{\widetilde{u}_{\widetilde{u}_{l}}} \ldots L_{\widetilde{u}_{\tilde{u}_{1}}}(\widetilde{h}) d \varphi_{j} \\
& \widetilde{\Theta}_{k}=\sum_{l=1}^{k-1} \sum_{\left(\widetilde{u}_{1}, \ldots, \widetilde{u}_{l}\right) \in \widetilde{\mathscr{U}}_{l}} g_{\widetilde{u}_{1} \ldots \widetilde{u}_{l}}(x) d L_{\widetilde{u}_{\widetilde{u}_{l}}} \ldots L_{\tilde{u}_{\widetilde{u}_{1}}}(\widetilde{h})+g_{k}(x) d \varphi_{q+1}
\end{aligned}
$$

with the property that $g_{\widetilde{u}_{1} \ldots \widetilde{u}_{l}}(),. g_{k}($.$) are \mathscr{C}^{\infty}$-functions which do not depend on $\left(\widetilde{X}_{1}, \ldots, \widetilde{X}_{q}\right)$.

\section{Proof of lemma 2}

- For $k=1$ :

Let $u_{1} \in \mathscr{U}_{1}$, by definition $\widetilde{\omega}_{\widetilde{u}_{1}}=d L_{\widetilde{u}_{1}}(\widetilde{h}) \wedge \tilde{\pi}$, and from claim 3 , we know that $i_{\widetilde{X}}\left(\widetilde{\omega}_{\widetilde{u}_{1}}\right)=d L_{\widetilde{u}_{1}}(\widetilde{h})-$ $\sum_{j=1}^{q+1} L_{\widetilde{X}_{j}} L_{\widetilde{u}_{1}}(\widetilde{h}) d \varphi_{j}=d L_{\widetilde{u}_{1}}(\widetilde{h})-\sum_{j=1}^{q} L_{\widetilde{X}_{j}} L_{\widetilde{u}_{1}}(\widetilde{h}) d \varphi_{j}+\Theta_{1}$, here $\Theta_{1}=L_{\widetilde{X}_{q+1}} L_{\widetilde{u}_{1}}(\widetilde{h}) d \varphi_{q+1}$. Hence (11), (12) are true for $k=1$.

- Assuming that (11), (12) hold for $1 \leq l \leq k-1$, and let us show them for $k$. Using the definition of $\widetilde{\omega}_{\widetilde{u}_{1} \ldots \widetilde{u}_{k}}$ and applying (12) for $k-1$, we get:

$$
\begin{aligned}
& \widetilde{\omega}_{\widetilde{u}_{1} \ldots \widetilde{u}_{k}}=d L_{\widetilde{u}_{\widetilde{u}_{k}}} \ldots L_{\widetilde{u}_{\widetilde{u}_{1}}}(\widetilde{h}) \wedge \tilde{\pi} \\
& -L_{\widetilde{u}_{k}}\left[\sum_{j=1}^{q} L_{\widetilde{X}_{j}} L_{\widetilde{u}_{k-1}} \ldots L_{\widetilde{u}_{1}}(\widetilde{h}) d \varphi_{j}\right] \wedge \tilde{\pi}+L_{\widetilde{u}_{\widetilde{u}_{k}}}\left(\Theta_{k-1}\right) \wedge \tilde{\pi} \\
& \Theta_{k-1}=\widetilde{\Theta}_{k-1}-\sum_{j=1}^{q} \sum_{l=1}^{k-2} \sum_{\left(\widetilde{u}_{1}, \ldots, \widetilde{u}_{l}\right) \in \widetilde{\mathscr{U}}_{l}} g_{\widetilde{u}_{1} \ldots \widetilde{u}_{l}}(x) L_{\widetilde{X}_{j}} L_{\widetilde{u}_{l}} \ldots \\
& L_{\widetilde{u}_{1}}(\widetilde{h}) d \varphi_{j} \\
& \widetilde{\Theta}_{k-1}=\sum_{l=1}^{k-2} \sum_{\left(\widetilde{u}_{1}, \ldots, \widetilde{u}_{l}\right) \in \widetilde{\mathscr{U}}_{l}} g_{\widetilde{u}_{1} \ldots \widetilde{u}_{l}}(x) d L_{{\widetilde{u_{l}}}_{l}} \ldots L_{\widetilde{u}_{\widetilde{u}_{1}}}(\widetilde{h}) \\
& +\widetilde{g}_{k-1}(x) d \varphi_{q+1}
\end{aligned}
$$

and $g_{\widetilde{u}_{1} \ldots \widetilde{u}_{l}}, \widetilde{g}_{k-1}$ do not depend on $\left(\widetilde{X}_{1}, \ldots, \widetilde{X}_{q}\right)$.

Using the fact that $d \varphi_{i} \in D_{v}$, for $1 \leq i \leq q$, and that $L_{f_{u}}\left(D_{v}\right) \subset D_{v}$, then the following equality holds for every smooth functions $a_{1}(x), \ldots, a_{q}(x)$ :

$$
L_{f_{u}}\left(\sum_{j=1}^{q} a_{j}(x) d \varphi_{j}\right) \wedge \tilde{\pi}=0
$$

Combining (14) with expressions of $\Theta_{k-1}, \widetilde{\Theta}_{k-1}$, we get:

$$
\begin{aligned}
& \widetilde{\omega}_{\widetilde{u}_{1} \ldots \widetilde{u}_{k}}=d L_{\widetilde{u}_{\widetilde{u}_{k}}} \ldots L_{\widetilde{u}_{\widetilde{u}_{1}}}(\widetilde{h}) \wedge \tilde{\pi}+\sum_{l=1}^{k-2} \sum_{\left(\widetilde{u}_{1}, \ldots, \widetilde{u}_{l}\right) \in \widetilde{\mathscr{U}}_{l}} L_{\widetilde{u}_{\widetilde{u}_{k}}} \\
& {\left[\widetilde{g}_{\widetilde{u}_{1} \ldots \widetilde{u}_{l}}(x) d L_{\widetilde{u}_{\widetilde{u}_{l}}} \ldots L_{\widetilde{u}_{\widetilde{u}_{1}}}(\widetilde{h})+\widetilde{g}_{k-1}(x) d \varphi_{q+1}\right] \wedge \tilde{\pi}}
\end{aligned}
$$


By construction $L_{\widetilde{u}_{\widetilde{u}_{k}}}\left[\widetilde{g}_{\widetilde{u}_{1} \ldots \widetilde{u}_{l}}(x) d L_{\widetilde{u}_{\widetilde{u}_{l}}} \ldots L_{\widetilde{u}_{\widetilde{u}_{1}}}(\widetilde{h})\right]$ and $L_{\widetilde{u}_{\tilde{u}_{k}}}\left(\widetilde{g}_{k-1}(x) d \varphi_{q+1}\right) \wedge \widetilde{\pi}$ do not depend on $\left(\widetilde{X}_{1}, \ldots, \widetilde{X}_{q}\right)$ and $\left\{d \varphi_{1}, \ldots, d \varphi_{q+1}\right\} \cup\left\{d L_{\widetilde{u}_{\widetilde{u}_{l}}} \ldots L_{{\widetilde{u_{1}}}_{1}}(\widetilde{h}) ;\left(\widetilde{u}_{1}, \ldots, \widetilde{u}_{l}\right) \in\right.$ $\left.\widetilde{\mathscr{U}_{l}}, 1 \leq l \leq k-1\right\}$ forms a basis of $\widetilde{D}_{k}$, hence the last term of the right hand expression (15) takes the form $\sum_{l=1}^{k-1} \sum_{\left(\widetilde{u}_{1}, \ldots, \widetilde{u}_{l}\right) \in \widetilde{\mathscr{U}}_{l}} g_{\widetilde{u}_{1} \ldots \widetilde{u}_{l}}(x) d L_{\widetilde{u}_{\widetilde{u}_{l}}} \ldots L_{\widetilde{u}_{\widetilde{u}_{1}}}(\widetilde{h}) \wedge \tilde{\pi}$, where the $g_{\widetilde{u}_{1} \ldots \widetilde{u}_{l}}(x)$ 's are $\mathscr{C}^{\infty}$-functions which do not depend on $\left(\widetilde{X}_{1}, \ldots, \widetilde{X}_{q}\right)$. Consequently, expression (11) is satisfied.

In order to end the proof of lemma 2, it remains only to check (12).

Applying claim 3 to expression (11), we get:

$$
\begin{aligned}
& i_{\widetilde{X}}\left(\widetilde{\omega}_{\widetilde{u}_{1} \ldots \widetilde{u}_{k}}\right)=d L_{f_{\tilde{u}_{k}}} \ldots L_{\tilde{u}_{\widetilde{u}_{1}}}(\widetilde{h})+\sum_{l=1}^{k-1} \sum_{\left(\widetilde{u}_{1}, \ldots, \widetilde{u}_{l}\right) \in \widetilde{\mathscr{U}}_{l}} g_{\widetilde{u}_{1} \ldots \widetilde{u}_{l}}(x) \\
& d L_{\tilde{u}_{\tilde{u}_{l}}} \ldots L_{\tilde{u}_{\tilde{u}_{1}}}(\widetilde{h})-\sum_{j=1}^{q+1} L_{\widetilde{X}_{j}} L_{f_{\tilde{u}_{k}}} \ldots L_{{\tilde{u_{1}}}_{\widetilde{u}_{1}}}(\widetilde{h}) d \varphi_{j} \\
& -\sum_{j=1}^{q+1 k-1} \sum_{\left(\widetilde{u}_{1}, \ldots, \widetilde{u}_{l}\right) \in \widetilde{\mathscr{U}}_{l}} g_{\widetilde{u}_{1} \ldots \widetilde{u}_{l}}(x) L_{\widetilde{X}_{j}} L_{\tilde{u}_{\widetilde{u}_{l}}} \ldots L_{\widetilde{u}_{\widetilde{u}_{1}}}(\widetilde{h}) d \varphi_{j}
\end{aligned}
$$

Finally, expression (12) follows from (16) in which we introduce:

$$
\begin{aligned}
& \widetilde{\Theta}_{k}=\sum_{l=1}^{k-1} \sum_{\left(\widetilde{u}_{1}, \ldots, \widetilde{u}_{l}\right) \in \widetilde{\widetilde{\mathscr{U}}_{l}}} g_{\widetilde{u}_{1} \ldots \widetilde{u}_{l}}(x) d L_{f_{\tilde{u}_{l}}} \ldots L_{f_{\tilde{u}_{1}}}(\widetilde{h})+g_{k}(x) d \varphi_{q+1} \\
& \text { where } g_{k}(x)=-L_{\widetilde{X}_{q+1}} L_{\widetilde{u}_{\tilde{u}_{k}}} \ldots L_{\tilde{f}_{\tilde{u}_{1}}}(\widetilde{h})-\sum_{l=1}^{k-1} \sum_{\left(\widetilde{u}_{1}, \ldots, \widetilde{u}_{l}\right) \in \widetilde{\mathscr{U}_{l}}} \\
& g_{\widetilde{u}_{1} \ldots \widetilde{u}_{l}}(x) L_{\widetilde{X}_{q+1}} L_{\widetilde{u}_{\tilde{u}_{l}}} \ldots L_{f_{\tilde{u}_{1}}}(\widetilde{h}) \\
& \left.\Theta_{k}=\widetilde{\Theta}_{k}-\sum_{j=1}^{q} \sum_{l=1}^{k-1} \sum_{\left(\widetilde{u}_{1}, \ldots, \widetilde{u}_{l}\right) \in \widetilde{\mathscr{U}}_{l}} g_{\widetilde{u}_{1} \ldots \widetilde{u}_{l}}(x) L_{\widetilde{X}_{j}} L_{\tilde{u}_{\tilde{u}_{l}}} \ldots L_{\tilde{u}_{\tilde{u}_{1}}} \widetilde{h}\right) d \varphi_{j}
\end{aligned}
$$

Moreover, by construction $g_{\widetilde{u}_{1} \ldots \widetilde{u}_{l}}(x)$ and $g_{k}$ do not depend on $\left(\widetilde{X}_{1}, \ldots, \widetilde{X}_{q}\right)$. This ends the proof of lemma 2.

Now we can state the algorithm which allows to calculate vector fields $X, \widetilde{X}_{1}, \ldots, \widetilde{X}_{q}, \widetilde{X}_{q+1}$ satisfying conditions 1$)$ and 2) of theorem 1.

Theorem 2: (Algorithm)

System (3) can be steered by a local change of coordinates around some $x^{0}$ to a cascade-observable system (1), if, and only if, the following conditions hold:

a) The flag of co-distributions $D_{0} \subset \ldots \subset D_{v}=D_{v+1}$, $\widetilde{D}_{0} / D_{v} \subset \ldots \subset \widetilde{D}_{\widetilde{v}} / D_{v}=\widetilde{D}_{\widetilde{v}+1} / D_{v}$ are of constant dimension on some neighborhood of $x^{0}$, and $\operatorname{dim}\left(D_{v}\right)=q$, $\operatorname{dim}\left(\widetilde{D}_{\widetilde{v}} / D_{\sharp}\right)=n-q$

b) Let $B_{v}$ and $\widetilde{B}_{\widetilde{v}}$ be any fixed bases of $D_{v} / D_{v-1}$ and $\widetilde{D}_{\widetilde{v}} / \widetilde{D}_{\widetilde{v}-1}$ (see the construction (8)). Let $Y$ and $\widetilde{Y}$ be any fixed vector fields of the form $[Y]=\left[Z_{u_{1}^{0} \ldots u_{v-1}^{0}}\right] \in B_{v}^{*}$ and $[\widetilde{Y}]=\left[\widetilde{Z}_{\widetilde{u}_{1}^{0} \ldots \widetilde{u}_{\widetilde{v}-1}^{0}}\right] \in \widetilde{B}_{\widetilde{v}}^{*}$, then the following properties hold:
1) The vector $X=(-1)^{v-1} Y^{u_{1}^{0} \ldots u_{v-1}^{0}}$ satisfies condition 1) of theorem 1.

2) Setting $\widetilde{X}_{q+1}=(-1)^{\widetilde{v}-1} \widetilde{Y}^{\widetilde{u}_{1}^{0} \ldots \widetilde{u}_{v}^{0}}$ and considering $\mathscr{C}^{\infty}$-functions $\varphi_{1}, \ldots, \varphi_{q+1}$ such that $\varphi_{1}=h, \varphi_{q+1}=\widetilde{h}$ and that $\left(d \varphi_{1}, \ldots, d \varphi_{q}\right)$ forms a basis of $i_{X}\left(\Omega^{X}\right)+$ $\mathbb{R} d h$. Let $\widetilde{X}_{1}, \ldots, \widetilde{X}_{q}$ be vector fields satisfying $L_{\widetilde{X}_{j}}\left(\varphi_{i}\right)=\delta_{i j}, 1 \leq j \leq q, 1 \leq i \leq q+1$, and such that for every $\left(\widetilde{u}_{1}, \ldots, \widetilde{u}_{k}\right) \in \widetilde{\mathscr{U}_{k}}, 1 \leq k \leq \widetilde{v}-1$, we have:

$$
\sum_{j=1}^{q} d\left(L_{\widetilde{X}_{j}} L_{\tilde{u}_{\tilde{u}_{k}}} \ldots L_{\widetilde{u}_{\widetilde{u}_{1}}}(\widetilde{h})\right) \wedge d \varphi_{j}=d \Theta_{\widetilde{u}_{1} \ldots \widetilde{u}_{k}}
$$

where $\Theta_{\widetilde{u}_{1} \ldots \widetilde{u}_{k}}$ is the one-differential form stated in (12). Then $\widetilde{X}_{1}, \ldots, \widetilde{X}_{q+1}$ satisfy condition 2 ) of theorem 1.

Remark 1: According to expression (12) of lemma 2, expression (18) is then equivalent to $d\left(i_{\widetilde{X}}\left(\widetilde{\omega}_{\widetilde{u}_{1} \ldots \widetilde{u}_{k}}\right)\right)=0$.

Some comments on the procedure of calculation of vector fields $X, \widetilde{X}_{1}, \ldots, \widetilde{X}_{q}, \widetilde{X}_{q+1}$ :

1) The calculation of the vector field $X$ requires only the knowledge of expressions of $f_{u}$ and $h$.

2) $\widetilde{X}_{q+1}$ can be directly computed from the knowledge of $X, f_{u}, h$ and $\widetilde{h}$.

3) For $1 \leq i \leq q+1$, the functions $\varphi_{i}$ can be deduced from $X, f_{u}$ and $h$ and $\widetilde{h}$.

4) Finally, we end these comments by giving the algorithm of computation of $\left(\widetilde{X}_{1}, \ldots, \widetilde{X}_{q}\right)$ :

Computation of $\left(\widetilde{X}_{1}, \ldots, \widetilde{X}_{q}\right)$ :

Based on the construction of $\widetilde{B}_{k}$ and the functions $\varphi_{1}, \ldots, \varphi_{q+1}$, the set $\left\{\varphi_{1}, \ldots, \varphi_{q+1}\right\} \cup\left\{L_{\tilde{u}_{\tilde{u}_{k}}} \ldots L_{\tilde{u}_{\tilde{u}_{1}}}(\widetilde{h}) ; 1 \leq\right.$ $\left.k \leq \widetilde{v}-1, \quad\left(\widetilde{u}_{1}, \ldots, \widetilde{u}_{k}\right) \in \widetilde{\mathscr{U}}_{k}\right\}$ forms a local system of coordinates, which we denote by $(\xi, \widetilde{\xi})$, and where

$$
\begin{gathered}
\xi=\left(\varphi_{1}, \ldots, \varphi_{q+1}\right)=\left(\xi_{1}, \ldots, \xi_{q+1}\right) \\
\widetilde{\xi}=\left(\widetilde{\xi}_{1}, \ldots, \widetilde{\xi}_{\widetilde{v}-1}\right), \quad \widetilde{\xi}_{k}=\left(\widetilde{\xi}_{k 1}, \ldots, \widetilde{\xi}_{k, \widetilde{d}_{k}}\right)
\end{gathered}
$$

where $\left\{d \widetilde{\xi}_{k 1}, \ldots, d \widetilde{\xi}_{k, \widetilde{d}_{k}}\right\}=\left\{d L_{\tilde{u}_{\tilde{u}_{k}}} \ldots L_{\tilde{u}_{\tilde{u}_{1}}}(\widetilde{h}) ;\left(\widetilde{u}_{1}, \ldots, \widetilde{u}_{k}\right) \in\right.$ $\left.\widetilde{\mathscr{U}_{k}}\right\}$, and $\left\{\left[d L_{\tilde{u}_{\tilde{u}_{k}}} \ldots L_{\tilde{u}_{\tilde{u}_{1}}}(\widetilde{h})\right] ;\left(\widetilde{u}_{1}, \ldots, \widetilde{u}_{k}\right) \in \widetilde{\mathscr{U}_{k}}\right\}=\widetilde{B}_{k+1}$.

Therefore, we adopt the following notations:

$$
\begin{gathered}
\widetilde{\omega}_{\widetilde{u}_{1} \ldots \widetilde{u}_{k}}=\widetilde{\omega}_{k i}=d \widetilde{\xi}_{k i} \\
L_{\widetilde{X}_{j}}\left(\widetilde{\xi}_{k i}\right)=L_{\widetilde{X}_{j}} L_{\widetilde{u}_{\widetilde{u}_{k}}} \ldots L_{\widetilde{u}_{\widetilde{u}_{1}}}(\widetilde{h})=\widetilde{X}_{k i}^{j}
\end{gathered}
$$

Using the fact that $L_{\widetilde{X}_{j}}\left(\varphi_{j}\right)=L_{\widetilde{X}_{j}}\left(\xi_{j}\right)=\delta_{i j}$, we obtain $\widetilde{X}_{j}=\frac{\partial}{\partial \xi_{j}}+\sum_{k=1}^{\widetilde{v}-1} \sum_{i=1}^{\widetilde{d}_{k}} \widetilde{X}_{k i}^{j} \frac{\partial}{\partial \widetilde{\xi}_{k i}}$. Thus, the expression (12) can be rewritten:

$$
i_{\widetilde{X}}\left(\widetilde{\omega}_{k i}\right)=d \widetilde{\xi}_{k i}-\sum_{j=1}^{q} \widetilde{X}_{k i}^{j} d \xi_{j}+\Theta_{k i}
$$

where the $\Theta_{k i}$ 's are one-differential forms depending at most on $\widetilde{X}_{l i}^{j}, 1 \leq l \leq k-1,1 \leq j \leq q+1$.

The calculation of $\widetilde{X}_{k i}^{j}$, s follows from the following recursive procedure: 
- We start by computing $\widetilde{X}_{1 i}^{j}, 1 \leq j \leq q, 1 \leq i \leq \widetilde{d}_{1}$ :

For $k=1$, expression (19) becomes:

$i_{\widetilde{X}}\left(\widetilde{\omega}_{1 i}\right)=d \widetilde{\xi}_{1 i}-\sum_{j=1}^{q} \widetilde{X}_{1 i}^{j} d \xi_{j}+\Theta_{1 i}$, where $\Theta_{1 i}$ is a known one-differential form which does not depend on $\widetilde{X}_{1}, \ldots, \widetilde{X}_{q}$. Now condition (18) of theorem 2 yields to:

$$
\sum_{j=1}^{q} d\left(\widetilde{X}_{1 i}^{j}\right) \wedge d \xi_{j}=d \Theta_{1 i}, \text { for } 1 \leq i \leq \widetilde{d}_{1}
$$

Hence the $\widetilde{X}_{1 i}^{j}$ 's follows from the simple PDE system:

$$
\begin{aligned}
& \text { for } 1 \leq j, l \leq q, 1 \leq i \leq \widetilde{d}_{1}, \\
& \frac{\partial \widetilde{X}_{1 i}^{j}}{\partial \xi_{l}}-\frac{\partial \widetilde{X}_{1 i}^{l}}{\partial \xi_{j}}=\theta_{1 i}^{j l} \\
& \text { for } 1 \leq j \leq q, 1 \leq i \leq \widetilde{d}_{1}, 1 \leq t \leq \widetilde{v}-1,1 \leq s \leq \widetilde{d}_{t}, \\
& \frac{\partial \widetilde{X}_{1 i}^{j}}{\partial \widetilde{\xi}_{t s}}=\widetilde{\theta}_{1 s i}^{j t}
\end{aligned}
$$

where $\theta_{1 i}^{j l}$ and $\widetilde{\theta}_{1 s i}^{j t}$ are known functions depending only on the known vector field $\widetilde{X}_{q+1}$.

- Assuming that for $1 \leq j \leq q, 1 \leq l \leq k-1,1 \leq i \leq \widetilde{d_{l}}$, the functions $\widetilde{X}_{l i}^{j}$ are calculated, and let us compute $\widetilde{X}_{k i}^{j}$, $1 \leq i \leq \widetilde{d}_{k}$.

As for the first step, using expressions (18), (19) it follows that:

$$
\sum_{j=1}^{q} d\left(\widetilde{X}_{k i}^{j}\right) \wedge d \xi_{j}=d \Theta_{k i}
$$

which implies:

$$
\begin{aligned}
& \text { for } 1 \leq j, l \leq q, 1 \leq i \leq \widetilde{d}_{k}, \\
& \frac{\partial \widetilde{X}_{k i}^{j}}{\partial \xi_{l}}-\frac{\partial \widetilde{X}_{k i}^{l}}{\partial \xi_{j}}=\theta_{k i}^{j l} \\
& \text { for } 1 \leq j \leq q, 1 \leq i \leq \widetilde{d}_{k}, 1 \leq t \leq \widetilde{v}-1,1 \leq s \leq \widetilde{d}_{t}, \\
& \frac{\partial \widetilde{X}_{k i}^{j}}{\partial \widetilde{\xi}_{t s}}=\widetilde{\theta}_{k s i}^{j t}
\end{aligned}
$$

where $\theta_{k i}^{j l}$ and $\widetilde{\theta}_{k s i}^{j t}$ are known functions depending on the computed functions $\widetilde{X}_{l i}^{j}, 1 \leq l \leq k-1$.

The proof of theorem 2 is based on the following proposition.

\section{Proposition 1:}

Assuming that system (1) is cascade-observable, then the following properties hold:

1) Let $[Y]=\left[Z_{u_{1} \ldots u_{v-1}}\right] \in B_{v}^{*}$ and $X=(-1)^{v-1} Y^{u_{1} \ldots u_{v-1}}$, then

$$
X=\frac{\partial}{\partial z_{1}}+\sum_{i=2}^{q} a_{i} \frac{\partial}{\partial z_{i}}+\sum_{i=1}^{n-q} b_{i}(z, \widetilde{z}) \frac{\partial}{\partial \widetilde{z}_{i}}
$$

where the $a_{i}$ 's are constants. Moreover, we have:

$$
i_{X}\left(\Omega^{X}\right)=i_{\frac{\partial}{\partial z_{1}}}\left(\Omega^{\frac{\partial}{\partial z_{1}}}\right) \operatorname{modulo}\left(\mathbb{R} d z_{1}\right)
$$

2) Let $[\widetilde{Y}]=\left[\widetilde{Z}_{\widetilde{u}_{1} \ldots \widetilde{u}_{\widetilde{v}-1}}\right]$ be an element of $\widetilde{B}_{\widetilde{v}}^{*}$ and $\widetilde{X}_{q+1}=$ $(-1)^{\widetilde{v}-1} \widetilde{Y}^{\widetilde{u}_{1} \ldots \widetilde{u}_{\widetilde{v}}-1}$, and setting $\varphi_{i}=z_{i}$, for $1 \leq i \leq q$ and $\varphi_{q+1}=\widetilde{z}_{1}$. Let $\left(\widetilde{X}_{1}, \ldots, \widetilde{X}_{q}\right)$ be a sequence of vector fields such that $L_{\widetilde{X}_{j}}\left(\varphi_{i}\right)=\delta_{i j}$ for $1 \leq j \leq q, 1 \leq i \leq q+1$ and satisfying condition (18) of theorem 2 , then:

$$
\widetilde{X}_{q+1}=\frac{\partial}{\partial \widetilde{z}_{1}}+\sum_{i=2}^{n-q} \widetilde{a}_{i} \frac{\partial}{\partial \widetilde{z}_{i}}
$$

where the $\widetilde{a}_{i}$ 's are constants and for $j=1, \ldots, q$,

$$
\widetilde{X}_{j}=\frac{\partial}{\partial z_{j}}+\sum_{i=2}^{n-q} \beta_{i j}(z, \widetilde{z}) \frac{\partial}{\partial \widetilde{z}_{i}}
$$

Moreover, if $\pi=d z_{1} \wedge \ldots \wedge d z_{q}, \widetilde{X}_{j}^{0}=\frac{\partial}{\partial z_{j}}$, for $1 \leq j \leq q$, $\widetilde{X}_{q+1}^{0}=\frac{\partial}{\partial \widetilde{z}_{1}}$, and $\widetilde{X}^{0}=\left(\widetilde{X}_{1}^{0}, \ldots, \ldots, \widetilde{X}_{q+1}^{0}\right)$, then,

$$
i_{\widetilde{X}}\left(\Omega_{\pi}^{\widetilde{X}}\right)=i_{\widetilde{X}^{0}}\left(\Omega_{\pi}^{\widetilde{X}^{0}}\right) \operatorname{modulo}\left(d \mathscr{A}_{q}+\mathbb{R} d \widetilde{z}_{1}\right)
$$

where $\mathscr{A}_{q}=\mathscr{C}^{\infty}\left\{z_{1}, \ldots, z_{q}\right\}$ stands for the ring of $\mathscr{C}^{\infty}$ functions of $\left(z_{1}, \ldots, z_{q}\right)$.

\section{Proof of proposition 1.}

Setting $A_{i}(u), \widetilde{A}_{i}(u)$ to be the respective $i$ th rows of $A(u)$, $\widetilde{A}(u)$, and $\psi_{i}(u, y), \widetilde{\psi}_{i}(u, z, \widetilde{y})$ are the $i$ th components of $\psi$ and $\widetilde{\psi}$. In the $(z, \widetilde{z})$-system of coordinates $f_{u}$ takes the form:

$f_{u}=\sum_{i=1}^{q}\left(A_{i}(u) z+\psi_{i}\left(u, z_{1}\right)\right) \frac{\partial}{\partial z_{i}}+\sum_{i=1}^{n-q}\left(\widetilde{A}_{i}(u) \widetilde{z}+\widetilde{\psi}_{i}\left(u, z, \widetilde{z}_{1}\right)\right) \frac{\partial}{\partial \widetilde{z}_{i}}$

Let $B_{k+1}=\left\{\left[d\left(L_{f_{u_{k}}} \ldots L_{f_{u_{1}}}\left(z_{1}\right)\right)\right] ;\left(u_{1}, \ldots, u_{k}\right) \in \mathscr{U}_{k}\right\}, \widetilde{B}_{k+1}=$ $\left\{\left[d\left(L_{\widetilde{u}_{k}} \ldots L_{\widetilde{u}_{1}}\left(\widetilde{z}_{1}\right)\right)\right] ; \quad\left(\widetilde{u}_{1}, \ldots, \widetilde{u}_{k}\right) \in \widetilde{\mathscr{U}}_{k}\right\}$ be the respective bases of $D_{k+1} / D_{k}$ and $\widetilde{D}_{k+1} / \widetilde{D}_{k}$.

From claim 1, we know that :

$$
\begin{aligned}
& {\left[d\left(L_{f_{u_{k}}} \ldots L_{f_{u_{1}}}\left(z_{1}\right)\right)\right]=\left[d C A\left(u_{1}\right) \ldots A\left(u_{l}\right) z\right]} \\
& {\left[d\left(L_{\widetilde{u}_{\widetilde{u}_{k}}} \ldots L_{\widetilde{u}_{1}}\left(\widetilde{z}_{1}\right)\right)\right]=\left[d \widetilde{C} \widetilde{A}\left(\widetilde{u}_{1}\right) \ldots \widetilde{A}\left(\widetilde{u}_{l}\right) \widetilde{z}\right]}
\end{aligned}
$$

and from claim 2, the flags of co-distributions $D_{0}=0 \subset$ $\ldots \subset D_{v}=D_{v+1} ; 0=\widetilde{D}_{0} / D_{v} \subset \ldots \subset \widetilde{D}_{\widetilde{v}} / D_{v}=\widetilde{D}_{\widetilde{v}+1} / D_{v}$ are of constant dimensions. Now setting $n_{k}=\operatorname{dim}\left(D_{k}\right), \widetilde{n}_{k}=$ $\operatorname{dim}\left(\widetilde{D}_{k} / D_{v}\right)$, then we have $n_{0}=0<n_{1}=1<\ldots<n_{v}=q$; $\widetilde{n}_{0}=0<\widetilde{n}_{1}=1<\ldots<\widetilde{n}_{\widetilde{v}}=n-q$. Moreover, after a $(z, \widetilde{z})-$ linear change of coordinates, it can be assumed that:

$$
\left\{\begin{aligned}
B_{k} & =\left(\left[d z_{1+n_{k-1}}\right], \ldots,\left[d z_{n_{k}}\right]\right) \\
\widetilde{B}_{k} & =\left(\left[d \widetilde{z}_{1+\widetilde{n}_{k-1}}\right], \ldots,\left[d \widetilde{z}_{\widetilde{n}_{k}}\right]\right)
\end{aligned}\right.
$$

and that in this new system of coordinates $A(u), \widetilde{A}(u)$ take the following triangular structure:

$\left\{\begin{array}{c}\text { for } 1 \leq k \leq v-1, \text { for } n_{k-1}+1 \leq i \leq n_{k}, \\ A_{i}(u) z=a_{i 1}(u) z_{1}+a_{i 2}(u) z_{2}+\ldots+a_{i, n_{k+1}}(u) z_{n_{k+1}}\end{array}\right.$

$\left\{\begin{array}{c}\text { for } 1 \leq k \leq \widetilde{v}-1, \text { for } \widetilde{n}_{k-1}+1 \leq i \leq \widetilde{n}_{k}, \\ \widetilde{A}_{i}(u) \widetilde{z}=\widetilde{a}_{i 1}(u) \widetilde{z}_{1}+\widetilde{a}_{i 2}(u) \widetilde{z}_{2}+\ldots+\widetilde{a}_{i, \widetilde{n}_{k+1}}(u) \widetilde{z}_{\widetilde{n}_{k+1}}\end{array}\right.$

- Proof of property 1) of proposition 1:

Proof of expression (22):

Let $Y=Z_{u_{1} \ldots u_{v-1}}$ be a fixed element of $B_{v}^{*}$ (the dual 
basis of $B_{v}$ ), thus after reordering $B_{v}$, it can be assumed that $\left[d L_{f_{u_{v-1}}} \ldots L_{f_{u_{1}}}\left(z_{1}\right)\right]=d z_{q}$, and hence:

$$
d z_{n_{v-1}+i}(Y)=L_{Y}\left(z_{n_{v-1}+i}\right)=\delta_{q i}, 1 \leq i \leq n_{v}-n_{v-1}
$$

Recalling that $B_{v}^{*}$ is a basis of $\Delta_{v-1} / \Delta_{V}$ and that $\Delta_{v} \subset$ $\Delta_{v-1} \subset \ldots$, and that $\Delta_{k}=\operatorname{Ker}\left(D_{k}\right)$. Combining this last fact with (33), it follows that:

$$
Y=\frac{\partial}{\partial z_{q}}+\sum_{i=1}^{n-q} \beta_{j}^{*}(z, \widetilde{z}) \frac{\partial}{\partial \widetilde{z}_{j}}
$$

Now setting $X=(-1)^{v-1} Y^{u_{1} \ldots u_{v-1}}$, and recalling that $Y^{u_{1} \ldots u_{v-1}}=\left[f_{u_{v-1}},\left[\ldots,\left[f_{u_{1}}, Y\right] \ldots\right]\right]$, then (27), (31), (34)) give rise to:

$$
Y^{u_{1} \ldots u_{v-1}}=\sum_{i=1}^{q} a_{i} \frac{\partial}{\partial z_{i}}+\sum_{i=1}^{n-q} b_{i}(z, \widetilde{z}) \frac{\partial}{\partial \widetilde{z_{i}}}
$$

where the $a_{i}$ 's are constants.

Finally, from lemma 1 , it follows that $d z_{1}\left(Y^{u_{1} \ldots u_{v-1}}\right)=-d L_{f_{u_{v-1}}}\left(z_{1}\right)\left(Y^{u_{1} \ldots u_{v-2}}\right)=\ldots=$ $(-1)^{v-1} d\left(L_{f_{u_{v-1}}} \ldots L_{f_{u_{1}}}\left(z_{1}\right)\right)(Y)=(-1)^{v-1} d z_{q}(Y)=$ $(-1)^{v-1}$, hence:

$X=(-1)^{v-1} Y^{u_{1} \ldots u_{v-1}}=\frac{\partial}{\partial z_{1}}+\sum_{i=2}^{q} a_{i} \frac{\partial}{\partial z_{i}}+\sum_{i=1}^{n-q} b_{i}(z, \widetilde{z}) \frac{\partial}{\partial \widetilde{z_{i}}}$

Proof of expression (23):

Recalling that $\Omega^{X}$ is the real vector space spanned by the 2-differential forms $\omega_{u_{1} \ldots u_{k}}=L_{f_{u_{k}}}\left(i_{X}\left(\omega_{u_{1} \ldots u_{k-1}}\right)\right) \wedge d z_{1}$, where $\omega_{u}=d L_{f_{u}}\left(z_{1}\right) \wedge d z_{1}=d(C A(u)) z \wedge d z_{1}$.

Using the expression of $X$ given in (36) and the fact that $i_{X}\left(d \varphi \wedge d z_{1}\right)=d \varphi-L_{X}(\varphi) d z_{1}$ (see claim 3), then a simple algebraic computation yields to:

$$
\begin{aligned}
& i_{X}\left(\Omega^{X}\right)+\mathbb{R} d z_{1}=\operatorname{Span}\left\{d C A\left(u_{1}\right) \ldots C A\left(u_{k}\right) z ; k \geq 1,\right. \\
& \left.u_{i} \in \mathbb{R}^{m}\right\}+\mathbb{R} d z_{1}
\end{aligned}
$$

In particular, expression (37) holds for $X=\frac{\partial}{\partial z_{1}}$, hence $i_{X}\left(\Omega^{X}\right)=i_{\frac{\partial}{\partial z_{1}}}\left(\Omega^{\frac{\partial}{\partial z_{1}}}\right) \operatorname{modulo}\left(\mathbb{R} d z_{1}\right)$.

- The proof of property 2) of proposition 1 can be obtained by following the same procedure as for the property 1).

\section{Proof of theorem 2.}

The sufficient condition is stated in theorem 1.

\section{Necessary condition:}

Since conditions a) and b) of theorem 2 are intrinsic (they do not depend on the system of coordinates), it suffices to check them for the cascade-observable system (1).

1) Condition a) of theorem 2 is a straightforward consequence of claim 2.

2) Condition b)-1) of theorem 2 :

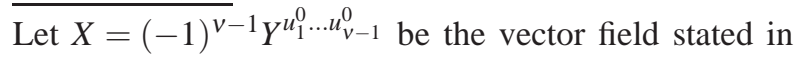
b)-1) of theorem 2, from (22)-(23) of proposition 1, we know that:

$$
\begin{aligned}
& X=\frac{\partial}{\partial z_{1}}+\sum_{i=2}^{q} a_{i} \frac{\partial}{\partial z_{i}}+\sum_{i=1}^{n-q} b_{i}(z, \widetilde{z}) \frac{\partial}{\partial \widetilde{z_{i}}} \\
& i_{X}\left(\Omega^{X}\right)=i_{\frac{\partial}{\partial z_{1}}}\left(\Omega^{\frac{\partial}{\partial z_{1}}}\right) \text { modulo }\left(\mathbb{R} d z_{1}\right)
\end{aligned}
$$

Combining (38) with the fact that $\frac{\partial}{\partial z_{1}}$ satisfies condition 1) of theorem 1 , it can be easily checked that $X$ meets conditions $1-i)$ to 1 )-iv) of theorem 1 . Hence condition b)-1) of theorem 2 is satisfied.

3) Condition b)-2) of theorem 2 :

Let $\widetilde{X}_{q+1}= \pm \widetilde{Y}^{0} \widetilde{u}_{1}^{0} \widetilde{u}_{\widetilde{v}-1}^{0}$ and considering linear functions $\varphi_{1}, \ldots, \varphi_{q+1}$ satisfying $\varphi_{1}=h=z_{1}, \varphi_{q+1}=\widetilde{h}=\widetilde{z}_{1}$ and such that $\left(d \varphi_{1}, \ldots, d \varphi_{q}\right)$ forms a basis of $i_{X}\left(\Omega^{X}\right)+$ $\mathbb{R} d z_{1}$. Up to a linear change of coordinates, it can be assumed that:

$$
\left(d \varphi_{1}, \ldots, d \varphi_{q}\right)=\left(d z_{1}, \ldots, d z_{q}\right)
$$

Now considering vector fields $\widetilde{X}_{1}, \ldots, \widetilde{X}_{q}$ such that $L_{\widetilde{X}_{j}}\left(\varphi_{i}\right)=\delta_{i j}, 1 \leq j \leq q, 1 \leq i \leq q+1$, and satisfying condition (18) of theorem 2. Namely, for every $\left(\widetilde{u}_{1}, \ldots, \widetilde{u}_{k}\right) \in \widetilde{\mathscr{U}_{k}}, 1 \leq k \leq \widetilde{v}-1$,

$$
\sum_{j=1}^{q} d\left(L_{\widetilde{X}_{j}} L_{\widetilde{u}_{\widetilde{u}_{k}}} \ldots L_{\widetilde{u}_{\widetilde{u}_{1}}}(\widetilde{h})\right) \wedge d \varphi_{j}=d \Theta_{\widetilde{u}_{1} \ldots \widetilde{u}_{k}}
$$

where $\Theta_{\widetilde{u}_{1} \ldots \widetilde{u}_{k}}$ is the one-differential form stated in (12). In order to check condition b)-2) of theorem 2, we will show that $\left(\widetilde{X}_{1}, \ldots, \widetilde{X}_{q+1}\right)$ meet condition 2) of theorem 1.

From 2) of proposition 1, we know that:

$$
\widetilde{X}_{q+1}=\frac{\partial}{\partial \widetilde{z}_{1}}+\sum_{i=2}^{n-q} \widetilde{a}_{i} \frac{\partial}{\partial \widetilde{z}_{i}}
$$

where the $\widetilde{a}_{i}$ 's are constants and for $j=1, \ldots, q$,

$$
\widetilde{X}_{j}=\frac{\partial}{\partial z_{j}}+\sum_{i=2}^{n-q} \beta_{i j}(z, \widetilde{z}) \frac{\partial}{\partial \widetilde{z}_{i}}
$$

Moreover, if we set $\pi=d z_{1} \wedge \ldots \wedge d z_{q}, \widetilde{X}_{j}^{0}=\frac{\partial}{\partial z_{j}}$, for $1 \leq j \leq q, \widetilde{X}_{q+1}^{0}=\frac{\partial}{\partial \widetilde{z}_{1}}$, and $\widetilde{X}^{0}=\left(\widetilde{X}_{1}^{0}, \ldots, \ldots, \widetilde{X}_{q+1}^{0}\right)$, then:

$$
i_{\widetilde{X}}\left(\Omega_{\pi}^{\widetilde{X}}\right)=i_{\widetilde{X}^{0}}\left(\Omega_{\pi}^{\widetilde{X}^{0}}\right) \operatorname{modulo}\left(d \mathscr{A}_{q}+\mathbb{R} d \widetilde{z}_{1}\right)
$$

where $\mathscr{A}_{q}=\mathscr{C}^{\infty}\left\{z_{1}, \ldots, z_{q}\right\}$.

Thus, we have:

- By construction, $L_{\widetilde{X}_{i}}\left(\varphi_{j}\right)=\delta_{i j}$, hence condition 2-i) of theorem 1 is satisfied.

- From the check of the proof of the necessary condition of theorem 1 given in the subsection II$\mathrm{B}$, we know that the $(q+1)$-tuple of vector fields $\left(\widetilde{X}_{1}^{0}, \ldots, \ldots, \widetilde{X}_{q+1}^{0}\right)$ meet conditions 2 -ii) of theorem 1 , namely, $\operatorname{dim}\left(\Omega_{\pi}^{\widetilde{X}^{0}}\right)=n-q-1$. Consequently, to show 
that $\left(\widetilde{X}_{1}, \ldots, \widetilde{X}_{q+1}\right)$ meet condition 2-ii) of theorem 1 $\left(\operatorname{dim}\left(\Omega_{\pi}^{\widetilde{X}}\right)=n-q-1\right)$, it suffices to show that:

$$
\Omega_{\pi}^{\widetilde{X}}=\Omega_{\pi}^{\widetilde{X}^{0}}
$$

By definition, $\Omega_{\pi}^{\widetilde{X}}=\sum_{u \in \mathbb{R}^{m}} L_{f_{u}}\left(i_{\widetilde{X}}\left(\Omega_{\pi}^{\widetilde{X}}\right)\right) \wedge d z_{1} \ldots \wedge$ $d z_{q} \wedge d \widetilde{z}_{1}$, where $f_{u}=\sum_{i=1}^{q}\left(A_{i}(u) z+\psi_{i}(u, y)\right) \frac{\partial}{\partial z_{i}}+$ $\sum_{i=1}^{n-q}\left(\widetilde{A}_{i}(u) \widetilde{z}+\widetilde{\psi}_{i}(u, z, \widetilde{y})\right) \frac{\partial}{\partial \widetilde{z}_{i}}$. Moreover, it is easy to see that $L_{f_{u}}\left(d \mathscr{A}_{q}\right) \wedge d z_{1} \ldots \wedge d z_{q} \wedge d \widetilde{z}_{1}=0$, thus $L_{f_{u}}\left(d \mathscr{A}_{q}+\mathbb{R} d \widetilde{z}_{1}\right) \wedge d z_{1} \ldots \wedge d z_{q} \wedge d \widetilde{z}_{1} \subset \mathbb{R} d(\widetilde{C} \widetilde{A}(u) \widetilde{z}) \wedge$ $d z_{1} \ldots \wedge d z_{q} \wedge d \widetilde{z}_{1} \subset \Omega_{\pi}^{\widetilde{X}^{0}}$. Combining this last fact with (43), we deduce (44).

- Condition 2-iii) of theorem 1 consists to verify that $d \widetilde{\omega}=0$, for every $\widetilde{\omega} \in i_{\widetilde{X}}\left(\Omega_{\pi}^{\widetilde{X}}\right)$. This property follows from the facts that $d\left(i_{\widetilde{X}^{0}}\left(\Omega_{\pi}^{\widetilde{X}^{0}}\right)\right)=0, d\left(d \mathscr{A}_{q}+\right.$ $\left.\left.\mathbb{R} d \widetilde{z}_{1}\right)\right)=0$ and (43).

- Condition 2-iv) of theorem 1:

It consists to show that the real vector space $\wedge^{n-q-1}\left(i_{\widetilde{X}}\left(\Omega_{\pi}^{\widetilde{X}}\right)\right) \wedge d \varphi_{1} \wedge \ldots \wedge d \varphi_{q+1}$ is of dimension 1.

According to (39) and (43), we have: $\wedge^{n-q-1}\left(i_{\widetilde{X}}\left(\Omega_{\pi}^{\widetilde{X}}\right)\right) \wedge d \varphi_{1} \wedge \ldots \wedge d \varphi_{q+1}=$ $\wedge^{n-q-1}\left(i_{\widetilde{X}^{0}}\left(\Omega_{\pi}^{\widetilde{X}^{0}}\right)\right) \wedge d z_{1} \wedge \ldots \wedge d z_{q} \wedge d \widetilde{z}_{1}$. From the check of the proof of the necessary condition of theorem 1 given in the subsection II-B, we know that $\widetilde{X}^{0}$ meets condition 2-iv) of theorem 1 . Hence $\wedge^{n-q-1}\left(i_{\widetilde{X}^{0}}\left(\Omega_{\pi}^{\widetilde{X}^{0}}\right)\right) \wedge d z_{1} \wedge \ldots \wedge d z_{q} \wedge d \widetilde{z}_{1}$ is of dimension 1. This ends the proof of theorem 2 .

\section{CONCLUSION}

Motivated by the existence of an observer design for a class of observable cascade systems. In this paper, we have characterized the class of nonlinear systems which can be transformed by a local change of coordinates to a cascade form. First, we have stated necessary and sufficient conditions. Next, we have derived an algorithm permitting to transform a nonlinear system into such cascade observable form. Its extension to general multi-output case is a difficult task and requires solving complex PDE.

\section{REFERENCES}

[1] G. Besanon, G. Bornard, and H. Hammouri. Observer synthesis for a class of nonlinear systems. European Journal of control, 2(3):176192, 1996.

[2] D. Boutat, A. Benali, H. Hammouri, and K. Busawon. New algorithm for observer error linearization with a diffeomorphism on the outputs. Automatica, 45(10):2187-2193, 2009.

[3] A. Glumineau, C.-H. Moog, and F. Plestan. New algebraic-geometric conditions for the linearization by input-output injection. IEEE Transactions on Automatic Control, 41(4):598-603, 1996.

[4] H. Hammouri and J.-P. Gauthier. Bilinearization up to output injection. Systems and Control Letters, 11(2):139-149, 1988.

[5] H. Hammouri and J.-P. Gauthier. The time-varying linearization up to output injection. Tampa, Florida, 1989. In Proceedings of the 28th IEEE Conference on Decision and Control.

[6] H. Hammouri and J.-P. Gauthier. Global time-varying linearization up to output injection. SIAM Journal on Control and Optimization, 30(6):1295-1310, 1992.
[7] H. Hammouri and M. Kinnaert. A new procedure for time-varying linearization up to output injection. Systems and Control Letters, 28(1):151-157, 1996.

[8] P. Jouan. The time-varying linearization up to output injection. SIAM Journal on Control and Optimization, 41(6):1756-1778, 2003.

[9] A.-J. Krener and A. Isidori. Linearization by output injection and nonlinear observers. Systems and Control Letters, 3(1):47-52, 1983.

[10] A.-J. Krener and W. Respondek. Nonlinear observers with linearizable error dynamics. SIAM Journal on Control and Optimization, 23(2):197-216, 1985.

[11] W. Lin and C.-I. Byrnes. Remarks on linearization of discrete time autonomous systems and nonlinear observer design. Systems and Control Letters, 25(1):31-40, 1995.

[12] F. Plestan and A. Glumineau. Linearization by generalized inputoutput injection. Systems and Control Letters, 31(2):115-128, 1997.

[13] X.-H. Xia and W.-B. Gao. Nonlinear observer design by observer error linearization. SIAM Journal on Control and Optimization, 27(1):199216, 1989. 